Check for updates

Cite this: Mater. Chem. Front. 2018, 2, 2263

Received 31st July 2018,

Accepted 10th October 2018

DOI: 10.1039/c8qm00375k

rsc.li/frontiers-materials

\section{Wavelength dependent nonlinear optical response of tetraphenylethene aggregation-induced emission luminogens $\uparrow$}

\author{
Jianbo Xiong, ${ }^{a}$ Xinyue Li, ${ }^{\text {bc }}$ Chunqing Yuan, ${ }^{b}$ Sergey Semin, ${ }^{c}$ Zhaoquan Yao, ${ }^{a}$ \\ Jialiang $X u^{* a}{ }^{*}$ Theo Rasing ${ }^{b}$ and Xian-He Bu (D) *ad
}

\begin{abstract}
Since the discovery of the striking aggregation-induced emission (AIE) phenomenon, tremendous efforts have been devoted to the exploration of brilliant organic solid-state light emitting materials and their underlying mechanistic theories. While AIE is well established in linear optics, the nonlinear optical (NLO) properties of the AIE luminogens (AIEgens) remain relatively unexplored. Here, we present a detailed study of the NLO properties of crystalline prototypical AlEgens based on tetraphenylethene (TPE). The results disclose a highly efficient NLO response of TPE crystals which is strongly dependent on the wavelength of the incident near infrared (NIR) light. TPE exhibits mainly two-photon excited fluorescence (TPF) upon excitation at $700 \mathrm{~nm}<\lambda<800 \mathrm{~nm}$, while it exhibits three-photon excited fluorescence (3PF) if pumped at $800 \mathrm{~nm}<\lambda<840 \mathrm{~nm}$. For pump wavelengths longer than $840 \mathrm{~nm}$, mainly second harmonic generation (SHG) is observed. The introduction of halogen atoms into the TPE backbone results in comparable NLO hyperpolarizabilities, but allows for future derivations and functionalizations. The exploration of the nonlinear optical properties of AlEgens might open a new avenue to a next generation of multifunctional organic and composite photonic materials and devices.
\end{abstract}

\section{Introduction}

The discovery of the exquisite aggregation induced emission (AIE) phenomenon ${ }^{1,2}$ has triggered an intense activity in the area of brilliant organic solid state light emitting materials. ${ }^{3-5}$ Substantial AIE luminogens (AIEgens) have been rationally designed, synthesized ${ }^{6-12}$ and widely applied in such diverse fields as organic light-emitting diodes (OLEDs), ${ }^{13}$ chemical/ biological sensing, ${ }^{14}$ lasing, ${ }^{15}$ bioimaging, ${ }^{16}$ etc. However, most of the work on the established AIEgens is focused on their linear optical properties. ${ }^{17}$ In contrast, the nonlinear optical (NLO) properties of the AIEgens have attracted less attention,

\footnotetext{
${ }^{a}$ School of Materials Science and Engineering, National Institute for Advanced Materials, Tianjin Key Laboratory of Metal and Molecule-Based Material Chemistry, Nankai University, Tianjin 300350, China.

E-mail: jialiang.xu@nankai.edu.cn, buxh@nankai.edu.cn

${ }^{b}$ School of Chemical Engineering and Technology, Tianjin University, Tianjin 300350, China

${ }^{c}$ Radboud University, Institute for Molecules and Materials (IMM), Heyendaalsweg 135, 6525AJ Nijmegen, The Netherlands

${ }^{d}$ State Key Laboratory of Elemento-Organic Chemistry, College of Chemistry, Nankai University, Tianjin 300071, China

$\dagger$ Electronic supplementary information (ESI) available: Crystallographic data (CIF), PXRD, TG analysis, crystal morphologies, and transition dipole calculations. CCDC 1837773-1837775. For ESI and crystallographic data in CIF or other electronic format see DOI: $10.1039 / \mathrm{c} 8 q \mathrm{qm} 00375 \mathrm{k}$
}

although light emitting materials with high NLO coefficients are of crucial importance for a wide range of applications in modern technologies, ranging from light frequency conversion, ${ }^{18}$ optical telecommunications, ${ }^{19}$ information processing, ${ }^{20}$ to data storage. ${ }^{21}$ Only a number of AIEgens have been demonstrated to exhibit NLO properties. Tang, He et al. studied higher-order NLO effects from a 2,3-bis(4-(phenyl(4-(1,2,2-triphenylvinyl)phenyl)amino)phenyl)fumaronitrile (TTF) AIEgen, ${ }^{22}$ which found applications in up-conversion near-infrared (NIR) bioimaging. ${ }^{23}$ However, most of these AIEgens are active in odd-order NLO effects. ${ }^{24,25}$ For highly efficient even-order NLO such as second harmonic generation (SHG), a strict non-centrosymmetric molecular arrangement in the aggregation state is required. ${ }^{26-28} \mathrm{SHG}$ is a frequency-doubling process in which two incident photons combined to generate one photon with doubled frequency and energy. ${ }^{26}$ The exploration of NLO properties based on the typical AIE molecules may broaden the applications of AIE material. We have recently reported a hexaphenylene based AIEgen, 4-DBpFO, showing second-order nonlinear optical two photon excited fluorescence (TPF) along with second-order nonlinear optical SHG. ${ }^{29}$ However, the emission enhancement factor $\alpha_{\text {AIE }}(<10)$ and the quantum yield $\Phi(\sim 36 \%)$ of 4 -DBpFO are relatively low, which might limit its practical applications.

Tetraphenylethene (TPE) is a prototypical AIEgen with a very high emission enhancement factor and a virtually unity 


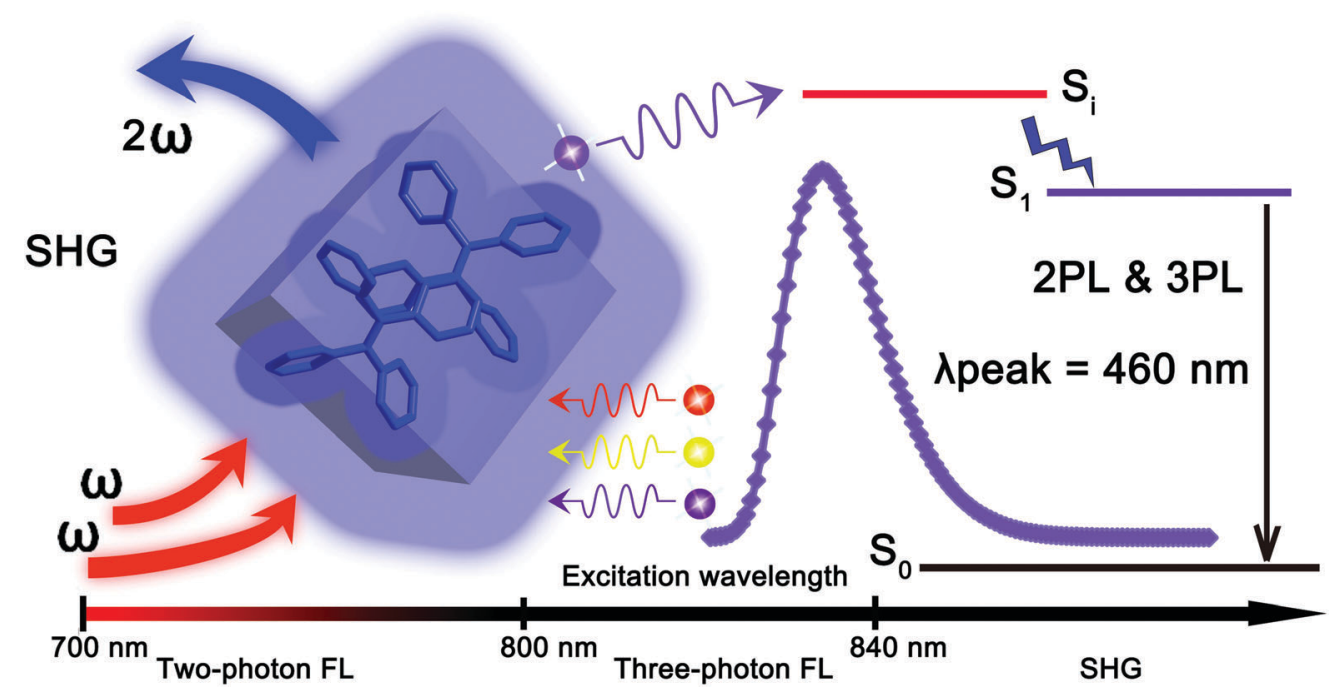

Scheme 1 Schematic representation of the wavelength-dependent NLO properties of TPE.

quantum yield in the solid state. ${ }^{30}$ The ease of its synthesis also offers diverse opportunities for molecularly engineered analogues with broad potential applications. It is also worth noting that TPE crystallizes in a $P_{21}$ space group, ${ }^{3,4,31,32}$ which is intrinsically polar and non-centrosymmetric. Such a noncentrosymmetric molecular arrangement might endow the TPE materials with highly efficient even-order NLO effects. So far, the NLO properties of model TPE AIEgens have not been explored yet. In this work, we studied in detail the NLO properties of TPE AIEgen and found that its microcrystals exhibit very different NLO effects when pumped with femtosecond (fs) laser pulses at different wavelengths. As shown in Scheme 1, the TPE crystal exhibits two-photon fluorescence, three-photon fluorescence and SHG when pumped with different incident beams of varied wavelengths. Importantly, the NLO signals of TPE show distinct wavelength dependent features. To be specific, when the pumped beam wavelength is larger than $840 \mathrm{~nm}$, mainly SHG is observed. 3PL (three-photon luminescence) emission is observed for incident wavelengths between $800 \mathrm{~nm}$ and $840 \mathrm{~nm}$, while only TPF is observed when the incident beam wavelength is shorter than $800 \mathrm{~nm}$.

Furthermore, comparable SHG coefficient with modified AIEgen building blocks were obtained by introducing $\mathrm{Br}$ and I halogen atoms to the TPE backbone. The halogenation of TPE with $\mathrm{Br}$ and I introduces new conjugated donor-acceptor and intramolecular charge transfer (ICT) ${ }^{33-35}$ effects may cause different transition dipole moment compared to TPE, the induced halogen-halogen effects together with the $\mathrm{C}-\mathrm{H} \cdots \pi$ interactions driving the crystalline system from monoclinic to orthogonal. In addition, the halogen active sites on TPE present possibilities for further design and modifications to obtain novel NLO AIEgens. We believe that the discovery of nonlinear optical (NLO) properties of these classic AIEgens may open a broad research area to explore new organic NLO materials and applications.

\section{Results and discussion}

\section{Wavelength dependent NLO properties of TPE microcrystals}

High-quality bulk single crystals of TPE were readily grown by slowly evaporating its saturated solution in a mixed solvent of THF and $n$-hexane (v/v, 1:1) at room temperature. The singlecrystal X-ray diffraction (SXRD) analysis suggests that the $\mathrm{X}$-shaped TPE molecules are organized in a non-centrosymmetric orthorhombic $P_{21}$ space group (point group: 2) (Table S1, ESI $\dagger$ ), in line with the structure reported in literature. ${ }^{32}$ Every unit cell contains two smallest asymmetrical TPE molecules, of which the four side phenyl planes are twisted from the central olefin by $\theta_{1}=45.6^{\circ}, \theta_{2}=55.0^{\circ}, \theta_{3}=46.5^{\circ}$ and $\theta_{4}=48.5^{\circ}$, respectively (Fig. $1 \mathrm{a}$ and b). These twists induce side $\mathrm{C}-\mathrm{H} \cdots \mathrm{C}$ contacts, driving the formation of molecular chains along the crystallographic $b$-axis, which are further linked into a three dimensional (3D) space structure by supramolecular interactions (Fig. S9a, ESI $\dagger$ ). The non-centrosymmetric nature of the TPE crystal endows its potential NLO properties. Indeed, the NLO spectra of TPE crystals exhibit strong SHG and multiple photon excitation fluorescence (Fig. 1c) upon fs laser excitations at different wavelengths. When the excitation wavelength is between $840 \mathrm{~nm}$ and $970 \mathrm{~nm}$, SHG signals with sharp peaks in the spectra are observed at half the wavelength of the corresponding incident light. However, when the excitation wavelength is lower than $840 \mathrm{~nm}$, a broad band emission centered around $460 \mathrm{~nm}$ appears in the spectra. Such a broad emission band is apparently an NLO process, considering its up-conversion characteristic from the NIR light to the visible range. Detailed power dependence measurements revealed the mechanism of these NLO processes (Fig. 1d). When the excitation beam wavelength is shorter than $800 \mathrm{~nm}$ (TPF) or greater than $840 \mathrm{~nm}$ (SHG), the slope of the power dependence of the intensity of the generated light is around 2.0, corroborating the two photon character of both processes. In contrast, a three-photon excitation character is observed with a slope around 3.0 for excitation wavelengths between $800 \mathrm{~nm}$ to $840 \mathrm{~nm} \cdot{ }^{36,37}$ The origin of the 
a)

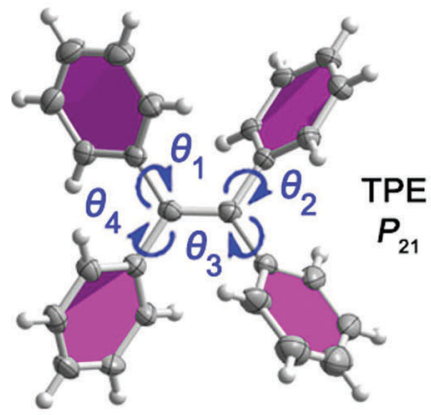

c)

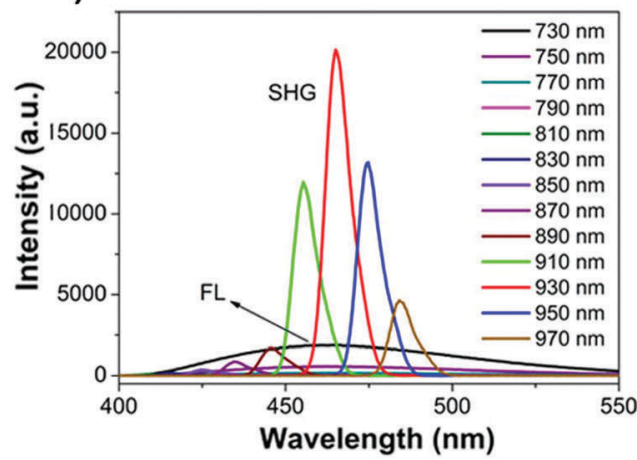

b)

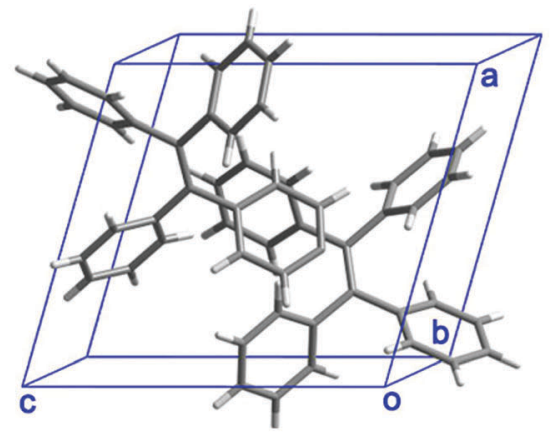

d)

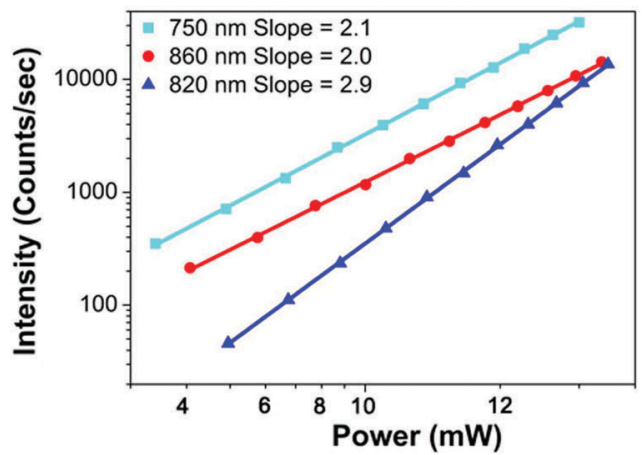

Fig. 1 (a) Molecular structure and torsion angles of TPE. (b) The unit cell structure of TPE. (c) The NLO spectra from a single TPE microcrystal pumped at different wavelengths ranging from 730 to $970 \mathrm{~nm}$, with an interval of $20 \mathrm{~nm}$, normalized by the square of the power of the pump at each wavelength. The broad peak centered at $460 \mathrm{~nm}$ is attributed to the two or three photon excited fluorescence, while the sharp peaks are from SHG. (d) Logarithmic plots of the power dependence of the generated NLO responses from the TPE microcrystal.

observed wavelength dependent phenomena can be attributed to the matching between the photon energy and the excitation energy threshold. That is, when exciting the TPE substrate with short wavelength light of substantial energy up to the energy gap between ground and excited state, the NLO process displays multi-photon fluorescence. ${ }^{22}$ In contrast, the NLO process shows an SHG signal when excited by long wavelength light with lower energy. Considering TPE is a prototype AIE molecule, the discovery of the wavelength dependent NLO properties may initiate a new way to explore NLO organic materials based on TPE AIEgens.

\section{Crystal structures and morphologies of halogenated TPEs}

Halogenation was performed on the initial TPE molecule. ${ }^{38,39}$ Both the resulting $4 \mathrm{Br}$-TPE and $4 \mathrm{I}$-TPE were found to crystallize in a non-centrosymmetric space group, while the formation of a non-centrosymmetric 4Cl-TPE supramolecular assembly failed. High quality crystals of 4Br-TPE and 4I-TPE suitable for single crystal XRD analysis were prepared by direct solvent evaporation of its solution in mixed THF and $n$-hexane. Very slow solvent evaporation resulted in large crystals with lengths in the centimeter scale (Fig. S4, ESI $\dagger$ ). The introduction of halogen atoms causes halogen-halogen interactions, ${ }^{40,41}$ which, in combination with the $\mathrm{C}-\mathrm{H} \cdots \pi$ interactions, drive the noncentrosymmetric crystallization of 4Br-TPE and 4I-TPE with the orthorhombic $P 22_{1} 2_{1} 2_{1}$ space group (point group: 222) (Table S1, ESI $\dagger$ ). Single crystal XRD analysis shows that each asymmetric unit contains four 4X-TPE (X = Br, I) molecules (Fig. S10, ESI $\dagger$ ). The twist angles between the four outstretched phenyl planes and the central olefin are $\theta_{1}=50.7^{\circ}, \theta_{2}=46.6^{\circ}, \theta_{3}=57.4^{\circ}$ and $\theta_{4}=51.2^{\circ}$ for $4 \mathrm{Br}-\mathrm{TPE}$, and $\theta_{1}=51.2^{\circ}, \theta_{2}=53.7^{\circ}, \theta_{3}=50.6^{\circ}$ and $\theta_{4}=49.0^{\circ}$ for $4 \mathrm{I}$-TPE. These twists can facilitate the $\mathrm{C}-\mathrm{H} \cdots \pi$ interactions. Double-chain structures of 4Br-TPE along the crystallographic $a$-axis are connected via these $\mathrm{C}-\mathrm{H} \cdots \pi$ interactions with distances of $3.1 \AA$ A. The synergistical $\mathrm{Br} \cdots \mathrm{Br}$ interactions with distances of $4.0 \AA$ is also found to stabilize the molecular chains along the $a$-axis. For $4 \mathrm{I}-\mathrm{TPE}$, similar double-chain structures along the $a$-axis are present with $\mathrm{C}-\mathrm{H} \cdots \pi$ distance $(3.1 \AA$ and $3.4 \AA$ ), while a molecular chain connected by $\mathrm{I} \cdots \mathrm{I}$ interactions ( $3.9 \AA$ ) is along the $b$-axis (Fig. S9, ESI $\dagger$ ). These multiple intermolecular interactions contribute to the molecular assembly and extend over the whole crystals. Furthermore, the simulation results on the morphologies of 4Br-TPE and 4I-TPE crystals agreed very well with the observed molecular and supramolecular selfassembled structures, confirming that the crystallographic $a$-axis ([001] direction) is the long axis of the microrods of both 4Br-TPE and 4I-TPE (Fig. 5).

It is important to clarify the relationship between the observed NLO signals and the supramolecular assembly from single molecules to microstructure architectures. Therefore, a clear and neat crystal morphology is necessary. ${ }^{37}$ The re-precipitation method was used to cultivate the micro-sized crystals of TPE and its derivatives in different morphologies and sizes. When the TPE micro crystal had a morphology of a diamond 

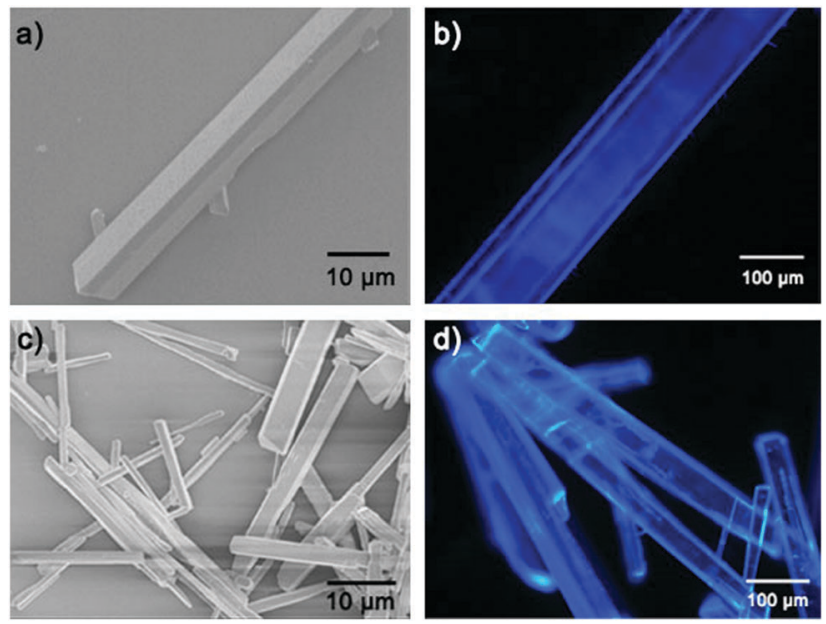

Fig. 2 Morphologies of 4Br-TPE and 4I-TPE microcrystals. (a) SEM image of microrod 4Br-TPE; (b) fluorescent microscopic image of 4Br-TPE; (c) SEM image of 4I-TPE microrod; (d) fluorescent microscopic image of 4I-TPE.

polyhedron (Fig. S1, ESI $\dagger$ ), nice rod-like microcrystals of both $4 \mathrm{Br}$-TPE and 4I-TPE have been grown by the re-precipitation method under the same conditions (Fig. 2). The well-defined 1D structure of these microcrystals paved the way for further elaborating the polarization-dependence of the NLO responses, allowing for the exploration of the relationships between the structure and the NLO properties. In a typical procedure, injecting a concentrated solution of 4Br-TPE or 4I-TPE in the effective solvent THF $\left(5 \mathrm{mg} \mathrm{mL}{ }^{-1}, 200 \mu \mathrm{L}\right)$ into the ineffective solvent $n$-hexane $(5 \mathrm{~mL})$ under vigorous stirring produces micro-sized rod-like crystals with widths of a few micrometers and lengths of tens of micrometers. Fluorescence microscope images (Fig. 2b and d) suggest that the microstructures of 4Br-TPE and 4I-TPE are both intense blue emitters under UV (330-380 nm) light excitation. Powder XRD patterns of the three compounds fitted well with their single-crystal XRD respectively, which further proved the crystal phase purity (Fig. S2, ESI $\dagger$ ). The TG analysis (Fig. S3, ESI $\dagger$ ) reveals that the halogenation significantly strengthens the stability of the TPE derivatives.

\section{Linear optical properties}

Emission spectrum of 4Br-TPE and 4I-TPE in solvents of diverse $\mathrm{H}_{2} \mathrm{O}$ /tetrahydrofuran (THF) gradients distinctively exhibit the AIE phenomenon (Fig. S5 and S6, ESI $\dagger$ ). The fluorescence intensity of both $4 \mathrm{Br}$-TPE and $4 \mathrm{I}$-TPE enhanced as the water fraction $\left(f_{\mathrm{w}}\right)$ increased gradually. ${ }^{42}$ The fluorescence intensity in absolute $\mathrm{H}_{2} \mathrm{O}$ were enhanced by nearly 14 and 15 times for 4Br-TPE and 4I-TPE in THF, respectively. Both the emission maximum of 4Br-TPE and 4I-TPE in the mixed solvents reveal a bathochromic shift tendency with the $f_{\mathrm{w}}$ increased from $0 \%$ to $90 \%$, and then hypsochromic shift to nearly the same as that of crystal states finally. This might be caused by the morphology
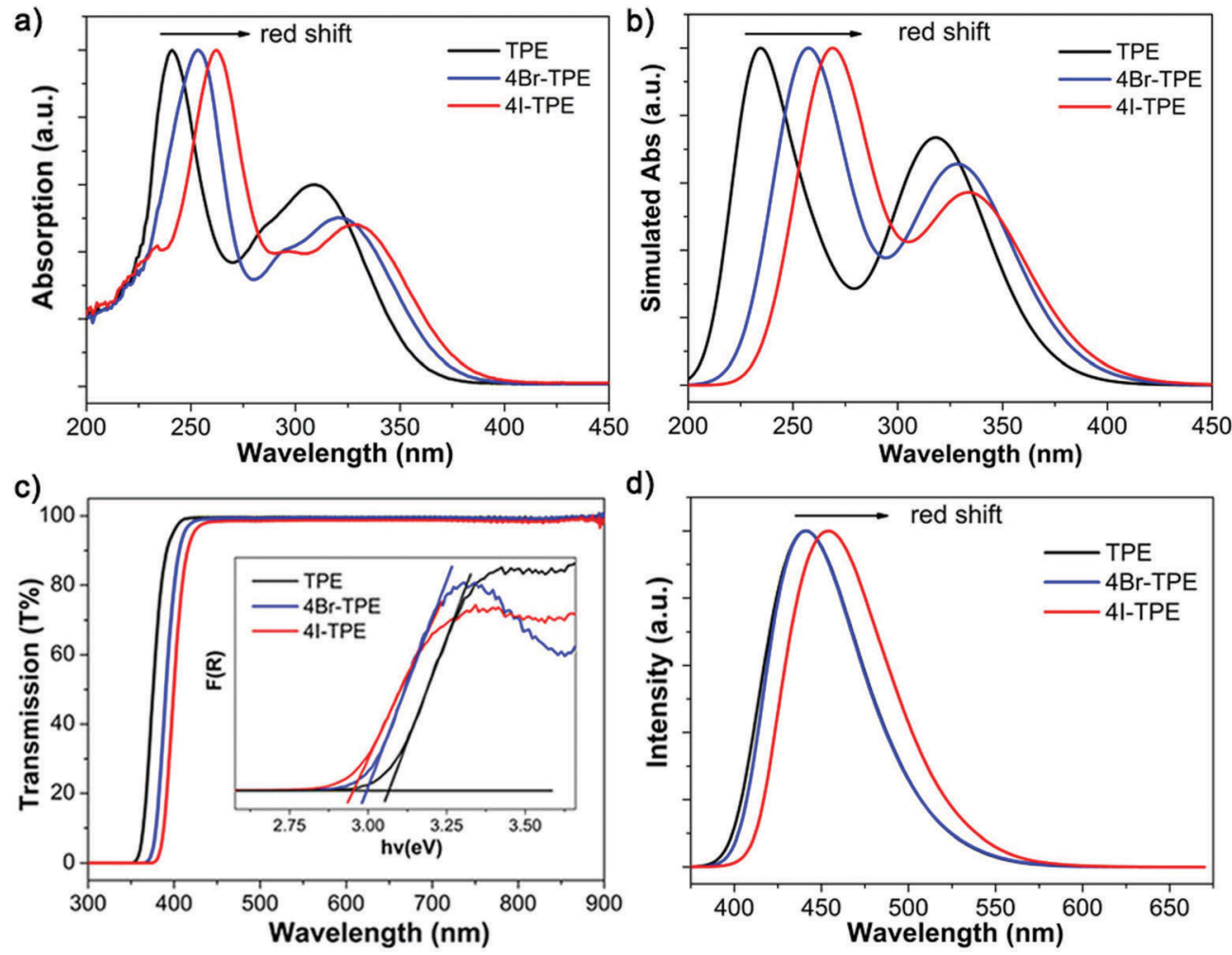

Fig. 3 Linear optical properties of 4Br-TPE and 4I-TPE, in comparison with those of TPE. (a) Normalized absorption spectra of TPE, 4Br-TPE and 4I-TPE in THF solution. (b) Simulated absorption spectra of TPE, 4Br-TPE and 4I-TPE in THF solution. (c) Transmission spectra of TPE, 4Br-TPE and 4I-TPE $\left(1 \times 10^{-3} \mathrm{M}, 1 \mathrm{~cm}\right.$ thickness). The inset shows the calculated band gaps of TPE, 4Br-TPE and 4I-TPE crystals based on the $(\alpha h \nu)^{2}-h \nu \mathrm{Curves}\left(\mathrm{TPE}: E_{\mathrm{g}}=3.06 \mathrm{eV}\right.$; 4Br-TPE: $E_{\mathrm{g}}=3.00 \mathrm{eV}$; 4I-TPE: $E_{\mathrm{g}}=2.95 \mathrm{eV}$ ). (d) Normalized photoluminescence spectra of crystallized TPE, 4Br-TPE and 4I-TPE. 
change of the aggregates. ${ }^{31}$ The AIE nature of $4 \mathrm{Br}$-TPE and 4I-TPE can be further prove by the temperature-dependent photoluminescence. As shown in Fig. S6 (ESI $\dagger$ ), the emission intensity in liquid nitrogen temperature $(77 \mathrm{~K}$ ) of $4 \mathrm{Br}-\mathrm{TPE}$ and 4I-TPE enhanced 4.6 and 6.6 times compared to that in the room temperature $(298 \mathrm{~K})$, respectively. That means the energy dissipation process is blocked at extremely low temperature. ${ }^{10}$ The linear optical properties of TPE, 4Br-TPE and 4I-TPE are shown in Fig. 3. The solid state photoluminescence (PL) spectra of TPE and 4Br-TPE exhibit emission bands both peaking at $440 \mathrm{~nm}$. The emission spectra of TPE coincide with the previously reported results ${ }^{3,4}$ and the PL spectra of 4Br-TPE shows a tiny red shift compared to that of TPE $(\sim 5 \mathrm{~nm})$. While the PL spectrum of 4I-TPE exhibits a distinct red shift $(14 \mathrm{~nm})$ with the emission peak centered at $454 \mathrm{~nm}$ (Fig. 3d). The crystal state absolute quantum yields $(\Phi)$ of $4 \mathrm{Br}-\mathrm{TPE}$ and $4 \mathrm{I}$-TPE were measured to be $30.07 \%$ and $15.49 \%$, respectively (Fig. S7 and S8, $\mathrm{ESI} \dagger)$. The reduction of quantum yield of $4 \mathrm{I}-\mathrm{TPE}$ compared to 4Br-TPE may induced by the heavy atom effect of iodine. ${ }^{43}$ The UV/vis diffuse reflectance spectra indicate that TPE and its halogenated derivatives have a wide transparent region with the absorption edges of 352,366 to $375 \mathrm{~nm}$ for TPE, 4Br-TPE and 4I-TPE, respectively (Fig. 3c). ${ }^{43}$ The measured absorption spectra of TPE, 4Br-TPE and 4I-TPE in THF display a red shift trend both on the primary band (visible region) and subordinated band (UV region) (Fig. 3a). Besides, the simulated absorption spectra exhibit the same red-shift tendency, in good agreement with the experimental results with slight difference less than $10 \mathrm{~nm}$ (Fig. 3b). The red-shift of the linear optical emission from TPE to 4I-TPE might be attributed to the heavy atom effect of the halogens. Specially, the excitations of the heavy halogen atoms have high

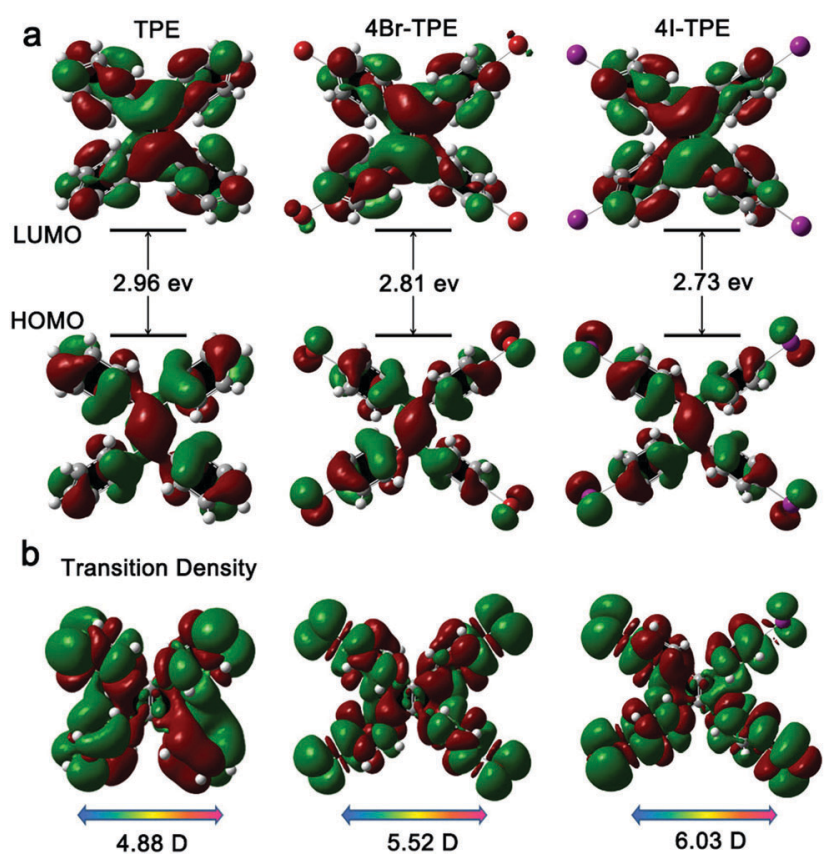

Fig. 4 (a) Calculated electronic structures and bandgaps of TPE, 4Br-TPE and 4I-TPE; (b) the transition densities corresponding to $S_{0}-S_{1}$ of TPE, 4Br-TPE and 4I-TPE. possibilities to undergo intersystem crossing transitions thus causing power reduction of the radiation. ${ }^{44}$ The band gaps as calculated from the crystal state absorption spectra also display a decreasing trend with values of 3.06, 3.00 and $2.95 \mathrm{eV}$ (Fig. 3c, inset).

In order to clarify the structure-property correlations, theoretical calculations of both the molecular and supramolecular structures were performed. Electronic structures of the three compounds show distinct charge-transfer characteristics between the highest occupied molecular orbitals (HOMOs) and the lowest unoccupied molecular orbitals (LUMOs) with band gaps of $2.96 \mathrm{eV}$, $2.81 \mathrm{eV}$ and $2.73 \mathrm{eV}$ for single molecules of TPE, 4Br-TPE and 4I-TPE, respectively (Fig. 4a). These values calculated based on crystal structures are $2.86 \mathrm{eV}, 2.71 \mathrm{eV}$ and $2.58 \mathrm{eV}$. The calculation results from both single molecules and aggregated crystal structures are nearly the same and accorded well with the bandgap values fitted from UV-vis absorption spectrum. ${ }^{4,46}$ The single molecule transition dipoles of the three compounds are calculated to be $4.88 \mathrm{D}, 5.52 \mathrm{D}$, and $6.03 \mathrm{D}$ for TPE, 4Br-TPE, and 4I-TPE, respectively (Fig. 4), and oriented vertical to the central olefin direction (Fig. S10 and S11, ESI $\dagger$ ). In contrast, the direction of the overall transition dipole of both $4 \mathrm{Br}-\mathrm{TPE}$ and $4 \mathrm{I}-\mathrm{TPE}$ is about $45^{\circ}$ offset from the long axis of the microrods ([001]; Fig. $5 \mathrm{c}$ and d).

\section{NLO properties of $4 \mathrm{Br}-\mathrm{TPE}$ and $4 \mathrm{I}-\mathrm{TPE}$}

The discovery of the wavelength-dependent NLO responses from TPE ignited our interest to further study the NLO properties of the

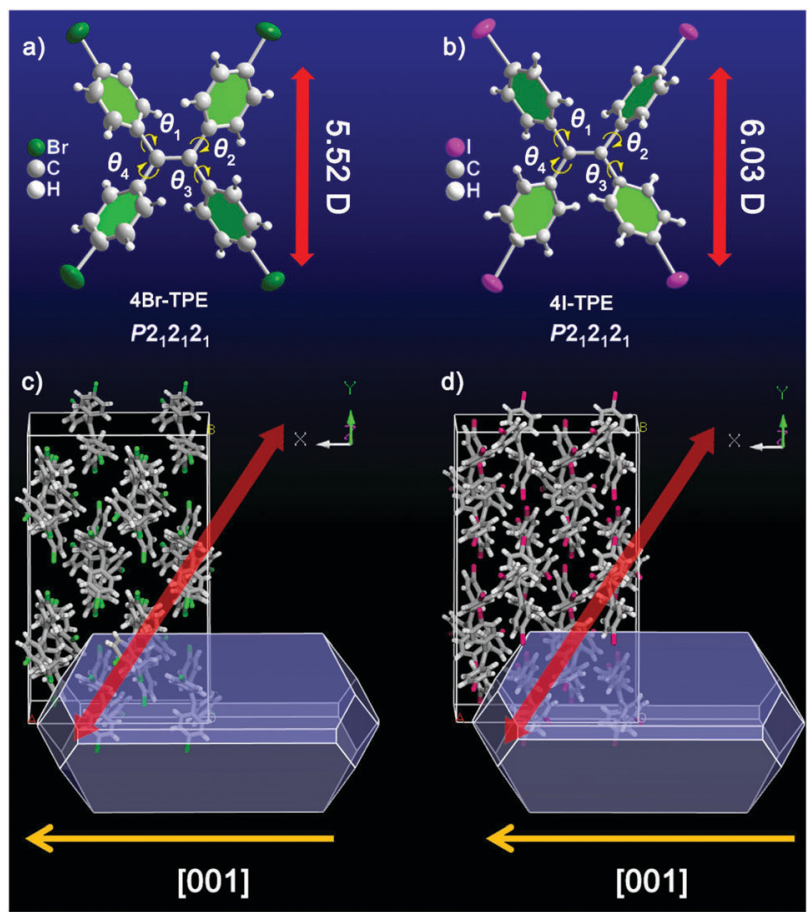

Fig. 5 Asymmetric molecular arrangement showing the torsion angles between the outstretched phenyl planes and central olefin of $4 \mathrm{Br}-\mathrm{TPE}$ (a) and 4I-TPE (b). The simulated morphology and supramolecular packing of $4 \mathrm{Br}$-TPE (c) and 4I-TPE (d), the inserted yellow arrows indicate the directions of the axis of the microrods, while the red arrows show the orientations of the overall transition dipole. 
halogenated TPE AIEgens (4Br-TPE and 4I-TPE) as they both crystallize in a non-centrosymmetric $P 2_{1} 2_{1} 2_{1}$ space group. As shown in Fig. 6a and b, the wavelength dependent NLO spectra vividly exhibit the frequency-doubling effect with the SHG peaks appearing at half the wavelength of the femtosecond excitation beam. The SHG signal of $4 \mathrm{Br}-\mathrm{TPE}$ can be tuned from 400 to $480 \mathrm{~nm}$ when the excitation beam was varied from 800 to $960 \mathrm{~nm}$, while the corresponding signals of 4I-TPE are tuned from 390 to $490 \mathrm{~nm}$ with the excitation from 780 to $980 \mathrm{~nm}$. The TPF peak of 4 I-TPE $(470 \mathrm{~nm})$ shows a red shift of $\sim 10 \mathrm{~nm}$ compared to that of $4 \mathrm{Br}-\mathrm{TPE}(460 \mathrm{~nm})$, which is mainly attributed to the heavy halogen atom effect of the iodine. ${ }^{47,48}$ It deserves to notice that for the crystal state of TPE, 4Br-TPE, and 4I-TPE, the pear position of TPF is red-shifted from their onephoton fluorescence, that is probably due to the reabsorption effect. $^{49}$ Similarly, the NLO emission of $4 \mathrm{Br}$-TPE and $4 \mathrm{I}-\mathrm{TPE}$ also posses wavelength dependent properties with mainly SHG signal when the wavelength of the excitation beam is longer than $800 \mathrm{~nm}$. TPF emission is observed when the excitation wavelength is between 740 and $800 \mathrm{~nm}$. The two-photon nature of the two NLO process is further demonstrated by the slope fitting of the relation between the NLO intensity and the pump laser power, which indicates a quadratic dependence (Fig. $6 \mathrm{c}$ and d). ${ }^{50}$
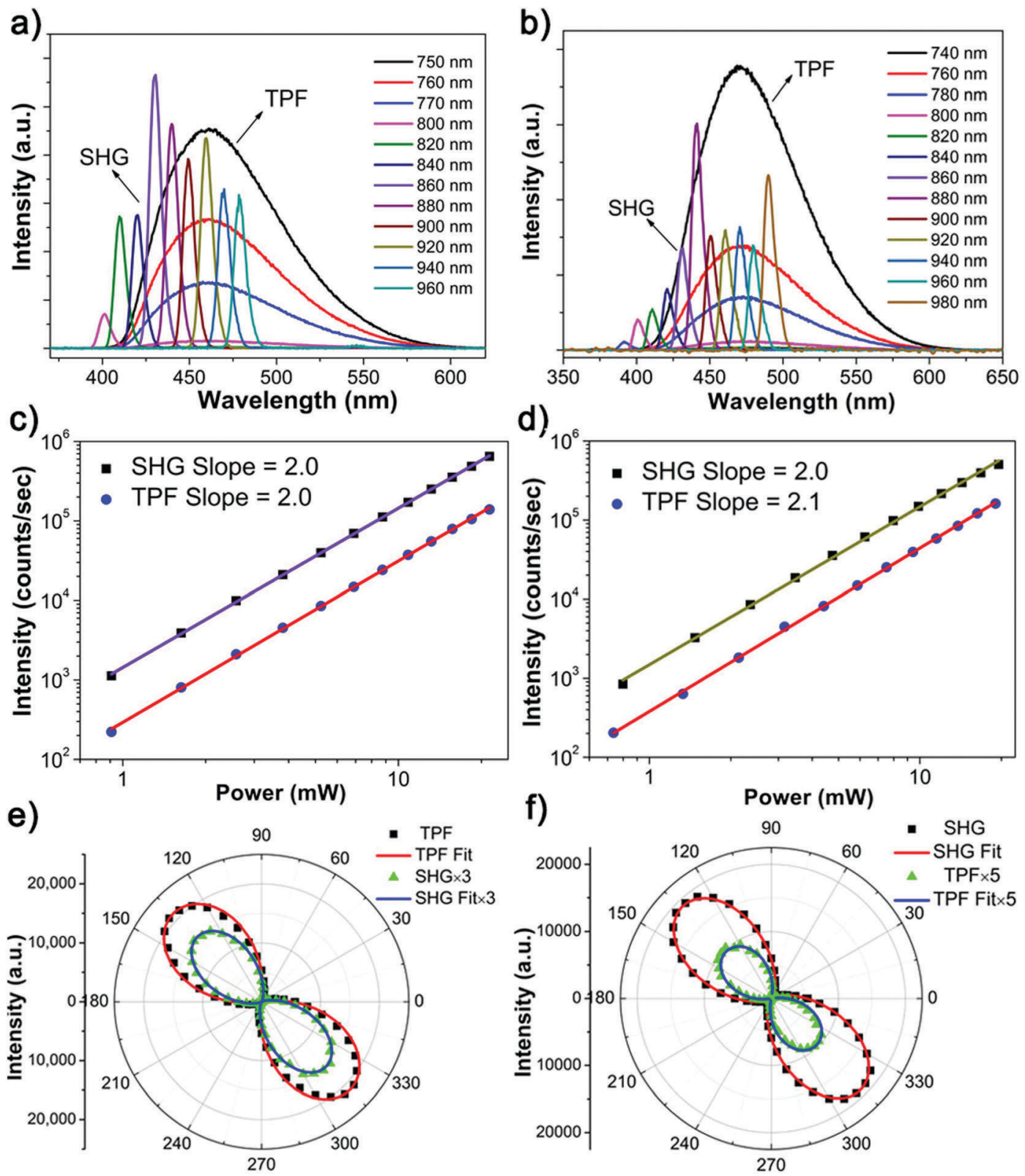

Fig. 6 Nonlinear optical properties of 4Br-TPE ( $a, c$ and e) and 4I-TPE (b, $d$ and f). The wavelength dependent NLO spectrum of 4Br-TPE (a) and 4I-TPE (b) pumped at different wavelengths ranging from 740 to $980 \mathrm{~nm}$, with an interval of $20 \mathrm{~nm}$, normalized by the square of the input laser power. The logarithmic fitting plot of the power dependence of SHG and TPF from 4Br-TPE (c) and 4I-TPE (d). Anisotropic SHG and TPF polarization dependent plots of $4 \mathrm{Br}$-TPE (e) and 4I-TPE (f), the red and blue lines indicate the $\cos ^{4} \theta$ and $\cos ^{2} \theta$ fits for SHG and TPF, respectively. 
The NLO responses from both the 4Br-TPE and 4I-TPE microrods were studied as function of the polarization of the incident light in order to clarify the anisotropic effects of the NLO signals. ${ }^{51}$ The microrod objects were aligned vertical to the plane of the polarized beam, and the polarization angle was tuned from $0^{\circ}$ to $180^{\circ} .^{37}$ As shown in Fig. 6e and f, both the SHG signal and TPF emission of 4Br-TPE and 4I-TPE have a similar polarization dependence trend. They both reach maxima when the polarization angle is near $135^{\circ}$ or $315^{\circ}$, which is about $45^{\circ}$ incline to the vertically aligned rodshaped crystal. The simulations suggest that the orientations of the $\mathrm{S}_{0}-\mathrm{S}_{1}$ transition dipoles of both $4 \mathrm{Br}-\mathrm{TPE}$ and $4 \mathrm{I}-\mathrm{TPE}$ are about $45^{\circ}$ deviated from the crystal long axis of the [001] direction, in good agreement with the measured polarization dependence. The polarization ratio of SHG, $\rho=\left(I_{\max }-I_{\min }\right) /\left(I_{\max }+I_{\min }\right)$, is $0.99 \pm$ 0.01 and $0.92 \pm 0.04$ for $4 \mathrm{Br}$-TPE and $4 \mathrm{I}$-TPE, while the polarization ratio of TPF is $0.95 \pm 0.04$ and $0.99 \pm 0.01$ for $4 \mathrm{Br}$-TPE and $4 \mathrm{I}-\mathrm{TPE}$, respectively. The SHG polarization ratio here is almost equally to that of DPFO (2,7-diphenyl-9H-fluoren-9-one), a typical NLO organic microfiber, while the TPF polarization ratio of halogenated TPE here are distinctly larger than that of DPFO. ${ }^{29}$ It is worth noting that the polarization ratios are practically one, which is higher than for the overwhelming majority of the reported organic crystals ${ }^{26,39,52}$ and even higher than some inorganic NLO materials. ${ }^{53}$ The TPF polarization ratio is more concerned to the transition dipole while the SHG polarization is mainly affected by permanent dipole moment, the bromine atoms contribute more to the permanent dipole moment, thus the 4I-TPE got the higher TPF polarization ratio and lower SHG polarization ratio compared to 4Br-TPE. The high polarization ratio values may be induced by the intramolecular charge transfer (ICT) ${ }^{33,48}$ between the aromatic benzenes and halogens, as well as the $\mathrm{S}_{0}-\mathrm{S}_{1}$ transition dipoles. ${ }^{54}$ The very high $\rho$ value and the nice agreement between the transition dipole simulations and the polarization dependent experiments further showed that the well-oriented transition dipole moment plays a vital role in the design of supramolecular AIE NLO materials.

\section{Conclusions}

The NLO response of the prototype AIEgen tetraphenylethylene (TPE) demonstrates various NLO properties, including SHG, TPF and 3PL depending on the wavelength of the excitation beam. Simple halogenation modification of the TPE AIEgen results in well-defined microrods of 4Br-TPE and 4I-TPE and comparable SHG signals with very high polarization ratios. Theoretical calculations together with polarization-dependent experiments show that the transition dipole moment is one of the key parameter for the design of NLO AIEgen materials with large hyperpolarizabilities. We believe this work may open a new area for the exploration of NLO materials based on AIEgens.

\section{Experimental}

\section{Reagents and materials}

All reagents were commercially purchased and used without further purification. Powder X-ray diffraction (PXRD) patterns were collected with $\mathrm{Cu} \mathrm{K} \alpha$ radiation $(\lambda=1.54178 \AA)$. Aboveroom-temperature thermo gravimetric analysis (TGA) measurements were made using a TA Instruments STD 2960 from 293 to 1000 K. Steady fluorescence spectra were collected on a Hitachi F-7000 fluorescence spectrophotometer. UV-VIS spectra and transmission spectra were recorded using a PERSEE TU-1950 UV-VIS spectrophotometer. The microcrystal morphology was characterized by SEM (FE-SEM, JSM-7800). Fluorescence images were obtained using an automated Leica DM5000B microscope equipped with a 340-380 $\mathrm{nm}$ band pass excitation filter cube. NLO examinations were performed using a homebuilt set-up. ${ }^{37}$ The calculations were conducted in Gaussian09 (Revision D.01) with Perdew-Burke-Ernzerhof (PBE) density function. ${ }^{55}$ The basis sets were employed lanl $2 \mathrm{dz}$ with pseudo potentials for $\mathrm{Br}$ and $\mathrm{I}$, and 6-31G(d) for other elements. The $k$-points for Brillouin zone were automatically selected as $4 \times 4 \times 4$ for TPE and $4 \times 4 \times 2$ for TPE-Br and TPE-I, respectively. The energy band gap of the crystals was represented by the minimal direct gap. The predicted crystal morphologies were simulated by materials studio 8.0 .

\section{Single-crystal X-ray crystallography}

Single-crystal X-ray diffraction data were collected on a SuperNova diffractometer with graphite-monochromated $\mathrm{Cu} \mathrm{K} \alpha$ radiation $(\lambda=1.54184 \AA)$. The structures were solved with direct methods using the SHELXTL program ${ }^{56}$ and refined with full-matrix least squares on $F^{2}{ }^{57}$ The corresponding Cambridge Crystallographic Data Centre (CCDC) reference number of TPE, 4Br-TPE and 4I-TPE are 1837773, 1837774 and 1837775, respectively. $\dagger$

\section{Conflicts of interest}

There are no conflicts to declare.

\section{Acknowledgements}

This work was supported by the National Natural Science Foundation of China (NSFC21531005, 91622111, and 21773168), "111 Project" of China (B18030), and the Netherlands Organization for Scientific Research (NWO VENI Grant, 680-47-437).

\section{Notes and references}

1 J. Luo, Z. Xie, J. W. Y. Lam, L. Cheng, B. Z. Tang, H. Chen, C. Qiu, H. S. Kwok, X. Zhan, Y. Liu and D. Zhu, Chem. Commun., 2001, 1740-1741.

2 B.-K. An, S.-K. Kwon, S.-D. Jung and S. Y. Park, J. Am. Chem. Soc., 2002, 124, 14410-14415.

3 J. Mei, Y. Hong, J. W. Lam, A. Qin, Y. Tang and B. Z. Tang, Adv. Mater., 2014, 26, 5429-5479.

4 J. Mei, N. L. Leung, R. T. Kwok, J. W. Lam and B. Z. Tang, Chem. Rev., 2015, 115, 11718-11940.

5 R. Hu, N. L. Leung and B. Z. Tang, Chem. Soc. Rev., 2014, 43, 4494-4562. 
6 M. Chen, L. Li, H. Nie, J. Tong, L. Yan, B. Xu, J. Z. Sun, W. Tian, Z. Zhao, A. Qin and B. Z. Tang, Chem. Sci., 2015, 6, 1932-1937.

7 T. Chen, Z.-Q. Chen, W.-L. Gong, C. Li and M.-Q. Zhu, Mater. Chem. Front., 2017, 1, 1841-1846.

8 Y. Cheng, J. Wang, Z. Qiu, X. Zheng, N. L. C. Leung, J. W. Y. Lam and B. Z. Tang, Adv. Mater., 2017, 29, 1703900.

9 H. Hamada, H. Tsuji and E. Nakamura, Mater. Chem. Front., 2018, 2, 296-299.

10 J. Xiong, K. Wang, Z. Yao, B. Zou, J. Xu and X.-H. Bu, ACS Appl. Mater. Interfaces, 2018, 10, 5819-5827.

11 J. Xu, L. Wen, W. Zhou, J. Lv, Y. Guo, M. Zhu, H. Liu, Y. Li and L. Jiang, J. Phys. Chem. C, 2009, 113, 5924-5932.

12 J. Xu, X. Liu, J. Lv, M. Zhu, C. Huang, W. Zhou, X. Yin, H. Liu, Y. Li and J. Ye, Langmuir, 2008, 24, 4231-4237.

13 H. Y. Chen, W. Y. Lam, J. D. Luo, Y. L. Ho, B. Z. Tang, D. B. Zhu, M. Wong and H. S. Kwok, Appl. Phys. Lett., 2002, 81, 574-576.

14 M. Wang, G. Zhang, D. Zhang, D. Zhu and B. Z. Tang, J. Mater. Chem., 2010, 20, 1858-1867.

15 Y. Hong, J. W. Y. Lam and B. Z. Tang, Chem. Commun., 2009, 4332-4353.

16 D. Ding, C. C. Goh, G. X. Feng, Z. J. Zhao, J. Liu, R. R. Liu, N. Tomczak, J. L. Geng, B. Z. Tang and L. G. Ng, Adv. Mater., 2013, 25, 6083-6088.

17 L. Feng, C. Wang, X. Deng, X. Miao, J. Wang, Y. Wang and Z. Li, Mater. Chem. Front., 2018, 2, 264-269.

18 D. Xiang and R. Gordon, ACS Photonics, 2016, 3, 1421-1425. 19 S. D. Smith, Nature, 1985, 316, 319.

20 D. Cotter, R. J. Manning, K. J. Blow, A. D. Ellis, A. E. Kelly, D. Nesset, I. D. Phillips, A. J. Poustie and D. C. Rogers, Science, 1999, 286, 1523-1528.

21 B. H. Cumpston, S. P. Ananthavel, S. Barlow, D. L. Dyer, J. E. Ehrlich, L. L. Erskine, A. A. Heikal, S. M. Kuebler, I. Y. S. Lee, D. McCord-Maughon, J. Q. Qin, H. Rockel, M. Rumi, X. L. Wu, S. R. Marder and J. W. Perry, Nature, 1999, 398, 51-54.

22 J. Qian, Z. Zhu, A. Qin, W. Qin, L. Chu, F. Cai, H. Zhang, Q. Wu, R. Hu, B. Z. Tang and S. He, Adv. Mater., 2015, 27, 2332-2339.

23 Z. Zhu, J. Qian, X. Zhao, W. Qin, R. Hu, H. Zhang, D. Li, Z. Xu, B. Z. Tang and S. He, ACS Nano, 2016, 10, 588-597.

24 R. Medishetty, V. Nalla, L. Nemec, S. Henke, D. Mayer, H. Sun, K. Reuter and R. A. Fischer, Adv. Mater., 2017, 29, 1605637.

25 J. Qi, C. Sun, D. Li, H. Zhang, We. Yu, A. Zebibula, J. W. Y. Lam, W. Xi, L. Zhu, F. Cai, P. Wei, C. Zhu, R. T. K. Kwok, L. L. Streich, R. Prevedel, J. Qian and B. Z. Tang, ACS Nano, 2018, 12, 7936-7945.

26 J. Xu, S. Semin, T. Rasing and A. E. Rowan, Small, 2015, 11, 1113-1129.

27 C. Wang, T. Zhang and W. Lin, Chem. Rev., 2012, 112, 1084-1104.

28 K. Pedersen, M. Schiek, J. Rafaelsen and H. G. Rubahn, Appl. Phys. B: Lasers Opt., 2009, 96, 821-826.

29 Y. Duan, C. Ju, G. Yang, E. Fron, E. Coutino-Gonzalez, S. Semin, C. Fan, R. S. Balok, J. Cremers, P. Tinnemans,
Y. Feng, Y. Li, J. Hofkens, A. E. Rowan, T. Rasing and J. Xu, Adv. Funct. Mater., 2016, 26, 8968-8977.

30 H. Yuan, K. Wang, K. Yang, B. Liu and B. Zou, J. Phys. Chem. Lett., 2014, 5, 2968-2973.

31 Z. Zhao, S. Chen, X. Shen, F. Maht, Y. Yu, P. Lu, J. W. Y. Lam, H. S. Kwoka and B. Z. Tang, Chem. Commun., 2010, 46, 686-688.

32 Y.-J. Jin, H. Kim, J. J. Kim, N. H. Heo, J. W. Shin, M. Teraguchi, T. Kaneko, T. Aoki and G. Kwak, Cryst. Growth Des., 2016, 16, 2804-2809.

33 G. Zhou, M. Baumgarten and K. Mullen, J. Am. Chem. Soc., 2008, 130, 12477-12484.

34 A. A. Fokin, O. Lauenstein, P. A. Gunchenko and P. R. Schreiner, J. Am. Chem. Soc., 2001, 123, 1842-1847.

35 A. Abdolmaleki, M. Dadsetani and A. Zabardasti, Spectrochim. Acta, Part A, 2018, 196, 353-365.

36 T. Chervy, J. Xu, Y. Duan, C. Wang, L. Mager, M. Frerejean, J. A. Munninghoff, P. Tinnemans, J. A. Hutchison, C. Genet, A. E. Rowan, T. Rasing and T. W. Ebbesen, Nano Lett., 2016, 16, 7352-7356.

37 J. Xu, S. Semin, D. Niedzialek, P. H. Kouwer, E. Fron, E. Coutino, M. Savoini, Y. Li, J. Hofkens, I. H. Uji, D. Beljonne, T. Rasing and A. E. Rowan, Adv. Mater., 2013, 25, 2084-2089.

38 H. Wang, B. Li, H. Wu, T. L. Hu, Z. Yao, W. Zhou, S. Xiang and B. Chen, J. Am. Chem. Soc., 2015, 137, 9963-9970.

39 F. M. A. Noa, S. A. Bourne and L. R. Nassimbeni, Cryst. Growth Des., 2015, 15, 3271-3279.

40 W. Z. Yuan, X. Y. Shen, H. Zhao, J. W. Y. Lam, L. Tang, P. Lu, C. Wang, Y. Liu, Z. Wang, Q. Zheng, J. Z. Sun, Y. Ma and B. Z. Tang, J. Phys. Chem. C, 2010, 114, 6090-6099.

41 Z. He, W. Zhao, J. W. Y. Lam, Q. Peng, H. Ma, G. Liang, Z. Shuai and B. Z. Tang, Nat. Commun., 2017, 8, 416.

42 J. Gong, P. Wei, Y. Sua, Y. Li, X. Feng, J. W. Y. Lam, D. Zhang, X. Song and B. Z. Tang, Chin. Chem. Lett., 2018, 29, 1493-1496.

43 D.-S. Zhang, Q. Gao, Z. Chang, X.-T. Liu, B. Zhao, Z.-H. Xuan, T.-L. Hu, Y.-H. Zhang, J. Zhu and X.-H. Bu, Adv. Mater., 2018, DOI: 10.1002/adma.201804715.

44 H.-T. Feng, X. Zheng, X. Gu, M. Chen, J. W. Y. Lam, X. Huang and B. Z. Tang, Chem. Mater., 2018, 30, 1285-1290.

45 H. Zhang, X. Zheng, N. Xie, Z. He, J. Liu, N. L. C. Leung, Y. Niu, X. Huang, K. S. Wong, R. T. K. Kwok, H. H. Y. Sung, I. D. Williams, A. Qin, J. W. Y. Lam and B. Z. Tang, J. Am. Chem. Soc., 2017, 139, 16264-16272.

46 J. Wang, X. Gu, P. Zhang, X. Huang, X. Zheng, M. Chen, H. Feng, R. T. K. Kwok, J. W. Y. Lam and B. Z. Tang, J. Am. Chem. Soc., 2017, 139, 16974-16979.

47 S. Gan, S. Hu, X.-L. Li, J. Zeng, D. Zhang, T. Huang, W. Luo, Z. Zhao, L. Duan, S.-J. Su and B.-Z. Tang, ACS Appl. Mater. Interfaces, 2018, 10, 17327-17334.

48 M. Z. K. Baig, P. K. Sahu, M. Sarkar and M. Chakravarty, J. Org. Chem., 2017, 82, 13359-13367.

49 G. S. He, L. S. Tan, Q. Zheng and P. N. Prasad, Chem. Rev., 2008, 108, 1245-1330.

50 C. K. Chen, A. R. B. de Castro and Y. R. Shen, Phys. Rev. Lett., 1981, 46, 145-148. 
51 S. Brasselet, V. Le Floc'h, F. Treussart, J. F. Roch, J. Zyss, E. Botzung-Appert and A. Ibanez, Phys. Rev. Lett., 2004, 92, 207401.

52 X. Li, S. Semin, L. A. Estrada, C. Yuan, Y. Duan, J. Cremers, P. Tinnemans, P. Kouwer, A. E. Rowan, T. Rasing and J. Xu, Chin. Chem. Lett., 2018, 29, 297-300.

53 J. C. Johnson, H. Yan, R. D. Schaller, P. B. Petersen, P. Yang and R. J. Saykally, Nano Lett., 2002, 2, 279-283.

54 J. Xu, S. Semin, J. Cremers, L. Wang, M. Savoini, E. Fron, E. Coutino, T. Chervy, C. Wang, Y. Li, H. Liu, Y. Li,
P. Tinnemans, P. H. J. Kouwer, T. W. Ebbesen, J. Hofkens, D. Beljonne, A. E. Rowan and T. Rasing, Adv. Opt. Mater., 2015, 3, 948-956.

55 J. P. Perdew, K. Burke and M. Ernzerhof, Phys. Rev. Lett., 1996, 77, 3865-3868.

56 G. M. Sheldrick, SHELXL97, Program for Crystal Structure Refinement, University of Göttingen, Göttingen, Germany, 1997.

57 A. L. Spek, J. Appl. Crystallogr., 2003, 36, 7-13. 